\title{
Supporting Information - Machine Learning Screening of Metal-ion Battery Electrode Materials
}

Isaiah A. Moses, ${ }^{\dagger}$ Rajendra P. Joshi, ${ }^{\ddagger}$ Burak Ozdemir, ${ }^{\top}$ Neeraj Kumar, ${ }^{\ddagger}$ Jesse Eickholt,,$^{\S}$ and Veronica Barone ${ }^{*, \|, \dagger}$

$\dagger$ Science of Advanced Materials Program, Central Michigan University, Mount Pleasant, MI 48859

$\ddagger$ Pacific Northwest National Laboratory, Richland, WA 99352, United States

9Department of Physics, Faculty of Science, University of Ostrava, 30. dubna 22, 70103 Ostrava, Czech Republic

$\S$ Department of Computer Science, Central Michigan University, Mount Pleasant, MI, 48859, USA

|| Department of Physics, Central Michigan University, Mount Pleasant, MI 48859

E-mail: v.barone@cmich.edu 


\section{Featurization}

From the chemical formulas of the electrodes and the matminer software $^{1}$ we obtained the features required to uniquely represent each of the pair of electrodes with low and high concentration of the working ion. The feature extraction modules used to transform the chemical compositions into representations suitable for machine learning are the ElementProperty, IonProperty, Stoichiometry, ValenceOrbital, BandCenter, and TMetalFraction. The modules implemented in the matminer are routines that have been developed to produce relevant descriptors for machine learning algorithms in order to correlate the descriptors with the target property we intend to predict. The ElementProperty gives the statistics of 22 different elemental properties. ${ }^{2}$ The statistics are the mean, mean absolute deviation, range, minimum, maximum and mode while the elemental properties are given in the Table S1. These properties are similar to those developed by Meredig et al. ${ }^{3}$ but the former has some additional properties and statistics which have made machine learning model built using the expanded attributes more accurate. ${ }^{2}$ Also adopted from the work of Ward et al. are IonProperty, Stoichiometry, and ValenceOrbital with the details elaborated below.

- IonProperty (3 features): This determines whether a compound is ionically bonded.

1. The first measure: This is a Boolean specifying whether a neutral ionic compound can be formed if each element takes exactly one of its common charge states.

2. The second measure: The maximum ionic character between any two elements in the material. The ionic character of every pair of elements A and B is computed using $I\left(X_{A}, X_{B}\right)=1-\exp -0.25\left(X_{A}-X_{B}\right)^{2}$ where $I$ is the fraction of ionic character, $X_{A}$ and $X_{B}$ are the electronegativity of element $A$ and $B$ respectively.

3. The third measure: The mean ionic character $\bar{I}=\sum\left(X_{i} X_{j} * I\left(X_{i}, X j\right)\right.$

- Stoichiometry (6 features): The number of type(s) of atom(s) in the chemical formula, and then the fraction of the elements present based on $L^{p}$ norms of a vector representing the atomic fraction of the material corresponding to each element given by: 
$\|x\|_{p}=\left(\sum_{i=0}^{n}\left|x_{i}\right|^{p}\right)^{1 / p}$ where $p=2,3,5,7,10$ are used. As an example,

$p=2$ norm for $\mathrm{TiO}_{2}$ is: $\quad\|x\|_{2}=\left(\left(\frac{1}{3}\right)^{2}+\left(\frac{2}{3}\right)^{2}\right)^{1 / 2}=0.7454$

$p=3$ norm for $\mathrm{TiO}_{2}$ is: $\quad\|x\|_{3}=\left(\left(\frac{1}{3}\right)^{3}+\left(\frac{2}{3}\right)^{3}\right)^{1 / 3}=0.6934$

- ValenceOrbital(4 features): The fraction-weighted average of the number of valence electrons in each orbital divided by the fraction-weighted average of the total number of valence electrons.

As an example, the fraction of $s$ electrons for $\mathrm{TiO}_{2}$ is $F_{s}=\frac{\frac{1}{2}(2)+\frac{2}{3}(2)}{\frac{1}{3}(4)+\frac{2}{3}(6)}=0.375$

Other properties include BandCenter and TMetalFraction, with the former providing the estimate of the absolute position of the band centre using the geometric mean of electronegativity $^{4}$ and the latter computing the fraction of magnetic transition metals. ${ }^{5}$ The total number of descriptors amounts to 306. These features, which are derived from the structure and chemical compositions of the electrodes, are expected to be sufficient to be applied to the ML algorithms to inform the correlation between the features and the target properties.

Table S1: The element properties for which statistics are used in the ElementProperty module. ${ }^{1,2}$

\begin{tabular}{|l|l|l|}
\hline Atomic number & Mendeleev number & Atomic weight \\
\hline Melting temperature & Column & Row \\
\hline Covalent radius & Electronegativity & $\# s$ valence electrons \\
\hline$\# p$ valence electrons & $\# d$ valence electrons & $\#$ valence electrons \\
\hline Total \# valence electrons & $\#$ unfilled $s$ states & \# unfilled $p$ states \\
\hline$\#$ unfilled $d$ states & \# unfilled $f$ states & Total \# unfilled states \\
\hline $\begin{array}{l}\text { Specific volume of 0 K } \\
\text { ground state }\end{array}$ & $\begin{array}{l}\text { Band gap energy of 0 K } \\
\text { ground state }\end{array}$ & $\begin{array}{l}\text { Magnetic moment } \\
\text { atom) of 0 K ground state }\end{array}$ \\
\hline $\begin{array}{l}\text { Space group number of 0 K } \\
\text { ground state }\end{array}$ & & \\
\hline
\end{tabular}




\section{Cross validation}

Table S2: The 10-fold cross validation. The training and validation data set (consisting of $90 \%$ of the available data) is divided into 10 folds, with each of the 10 folds labeled fold= $1,2,3, \ldots, 10$. The first round $\left(\mathrm{R}_{1}\right)$ takes the first fold as the validation data set $(\mathrm{V})$ whereas the remaining 9 folds are used for training $(\mathrm{T})$. This process is repeated for another 9 rounds $\left(\mathrm{R}_{2}\right.$ to $\left.\mathrm{R}_{10}\right)$. For each $\mathrm{R}_{i}$, the corresponding fold $i$ is used for validation and the remaining folds are used for training. The mean absolute error, $M A E$, is computed for each round $\mathrm{R}_{i}$ and the average of the $M A E$ for the 10 rounds of training, $\overline{M A E}$, is then calculated.

\begin{tabular}{l|cccccccccc|l}
\hline fold & 1 & 2 & 3 & 4 & 5 & 6 & 7 & 8 & 9 & 10 & \\
\hline $\mathrm{R}_{1}$ & $\mathrm{~V}$ & $\mathrm{~T}$ & $\mathrm{~T}$ & $\mathrm{~T}$ & $\mathrm{~T}$ & $\mathrm{~T}$ & $\mathrm{~T}$ & $\mathrm{~T}$ & $\mathrm{~T}$ & $\mathrm{~T}$ & $M A E_{1}$ \\
$\mathrm{R}_{2}$ & $\mathrm{~T}$ & $\mathrm{~V}$ & $\mathrm{~T}$ & $\mathrm{~T}$ & $\mathrm{~T}$ & $\mathrm{~T}$ & $\mathrm{~T}$ & $\mathrm{~T}$ & $\mathrm{~T}$ & $\mathrm{~T}$ & $M A E_{2}$ \\
$\mathrm{R}_{3}$ & $\mathrm{~T}$ & $\mathrm{~T}$ & $\mathrm{~V}$ & $\mathrm{~T}$ & $\mathrm{~T}$ & $\mathrm{~T}$ & $\mathrm{~T}$ & $\mathrm{~T}$ & $\mathrm{~T}$ & $\mathrm{~T}$ & $M A E_{3}$ \\
$\mathrm{R}_{4}$ & $\mathrm{~T}$ & $\mathrm{~T}$ & $\mathrm{~T}$ & $\mathrm{~V}$ & $\mathrm{~T}$ & $\mathrm{~T}$ & $\mathrm{~T}$ & $\mathrm{~T}$ & $\mathrm{~T}$ & $\mathrm{~T}$ & $M A E_{4}$ \\
$\mathrm{R}_{5}$ & $\mathrm{~T}$ & $\mathrm{~T}$ & $\mathrm{~T}$ & $\mathrm{~T}$ & $\mathrm{~V}$ & $\mathrm{~T}$ & $\mathrm{~T}$ & $\mathrm{~T}$ & $\mathrm{~T}$ & $\mathrm{~T}$ & $M A E_{5}$ \\
$\mathrm{R}_{6}$ & $\mathrm{~T}$ & $\mathrm{~T}$ & $\mathrm{~T}$ & $\mathrm{~T}$ & $\mathrm{~T}$ & $\mathrm{~V}$ & $\mathrm{~T}$ & $\mathrm{~T}$ & $\mathrm{~T}$ & $\mathrm{~T}$ & $M A E_{6}$ \\
$\mathrm{R}_{7}$ & $\mathrm{~T}$ & $\mathrm{~T}$ & $\mathrm{~T}$ & $\mathrm{~T}$ & $\mathrm{~T}$ & $\mathrm{~T}$ & $\mathrm{~V}$ & $\mathrm{~T}$ & $\mathrm{~T}$ & $\mathrm{~T}$ & $M A E_{7}$ \\
$\mathrm{R}_{8}$ & $\mathrm{~T}$ & $\mathrm{~T}$ & $\mathrm{~T}$ & $\mathrm{~T}$ & $\mathrm{~T}$ & $\mathrm{~T}$ & $\mathrm{~T}$ & $\mathrm{~V}$ & $\mathrm{~T}$ & $\mathrm{~T}$ & $M A E_{8}$ \\
$\mathrm{R}_{9}$ & $\mathrm{~T}$ & $\mathrm{~T}$ & $\mathrm{~T}$ & $\mathrm{~T}$ & $\mathrm{~T}$ & $\mathrm{~T}$ & $\mathrm{~T}$ & $\mathrm{~T}$ & $\mathrm{~V}$ & $\mathrm{~T}$ & $M A E_{9}$ \\
$\mathrm{R}_{10}$ & $\mathrm{~T}$ & $\mathrm{~T}$ & $\mathrm{~T}$ & $\mathrm{~T}$ & $\mathrm{~T}$ & $\mathrm{~T}$ & $\mathrm{~T}$ & $\mathrm{~T}$ & $\mathrm{~T}$ & $\mathrm{~V}$ & $M A E_{10}$ \\
\hline & & & & & & & & & & & $M A E$
\end{tabular}

\section{Data sets employed}

Table S3: Data instances used for training and validation $(\mathrm{T} \& \mathrm{~V})$ and testing for the different models using different data sets; All, Li, and Alkali. Different models are trained with both, data including all instances (Whole) and data after removing the underrepresented instances (Reduced), respectively.

\begin{tabular}{l|cc|cc|cc}
\hline & \multicolumn{2}{|c|}{ Whole } & \multicolumn{3}{c}{ Reduced } \\
\hline & \multicolumn{2}{|c|}{$\mathrm{V}_{a v}$ and $\Delta V_{\%}$ Models } & \multicolumn{2}{c}{$\mathrm{V}_{a v}$ Models } & $\Delta V_{\%}$ & Models \\
\hline Data set & $\mathrm{T} \& \mathrm{~V}$ & Testing & $\mathrm{T} \& \mathrm{~V}$ & Testing & $\mathrm{T} \& \mathrm{~V}$ & Testing \\
\hline All & 4371 & 485 & 4218 & 468 & 4192 & 465 \\
Li & 2324 & 258 & 2286 & 253 & 2310 & 256 \\
Alkali & 2884 & 320 & 2819 & 313 & 2801 & 311 \\
\hline
\end{tabular}




\section{Assessment}

Table S4: Comparison between experimental and ML average voltages, $\mathrm{V}_{a v}$, obtained from

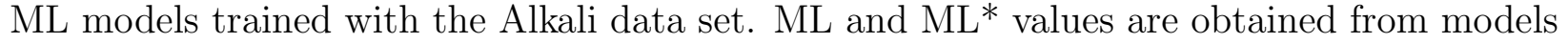
trained without underrepresented instances and with these instances included, respectively. Abs. dev. represent the absolute deviation between ML models and experiments.

\begin{tabular}{|c|c|c|c|c|c|c|}
\hline \multirow{2}{*}{\multicolumn{2}{|c|}{ Reaction }} & \multicolumn{3}{|c|}{$\mathbf{V}_{a v}(\mathbf{V})$} & \multicolumn{2}{|c|}{ Abs. dev.(V) } \\
\hline & & experiment & ML & ML* & ML & ML* \\
\hline 1 & $\mathrm{MnPO}_{4} \longleftrightarrow \mathrm{LiMnPO}_{4}$ & $4.1^{6}$ & 3.88 & 3.79 & 0.22 & 0.31 \\
\hline 2 & $\mathrm{FePO}_{4} \longleftrightarrow \mathrm{LiFePO}_{4}$ & $3.5^{7}$ & 3.22 & 3.78 & 0.28 & 0.28 \\
\hline 3 & $\mathrm{CoPO}_{4} \longleftrightarrow \mathrm{LiCoPO}_{4}$ & $4.8^{8}$ & 4.13 & 4.13 & 0.67 & 0.67 \\
\hline 4 & $\mathrm{LiCo}_{2} \mathrm{O}_{4} \longleftrightarrow \mathrm{Li}_{2} \mathrm{Co}_{2} \mathrm{O}_{4}$ & $3.5^{9}$ & 2.94 & 3.34 & 0.56 & 0.16 \\
\hline 5 & $\mathrm{NiO}_{2} \longleftrightarrow \mathrm{LiNiO}_{2}$ & $3.85^{10}$ & 3.55 & 3.57 & 0.30 & 0.28 \\
\hline 6 & $\mathrm{Mn}_{2} \mathrm{O}_{4} \longleftrightarrow \mathrm{LiMn}_{2} \mathrm{O}_{4}$ & $4.15^{11}$ & 4.32 & 3.94 & 0.17 & 0.21 \\
\hline 7 & $\mathrm{LiMn}_{2} \mathrm{O}_{4} \longleftrightarrow \mathrm{Li}_{2} \mathrm{Mn}_{2} \mathrm{O}_{4}$ & $2.95^{11}$ & 2.68 & 3.08 & 0.27 & 0.13 \\
\hline 8 & $\mathrm{CoO}_{2} \longleftrightarrow \mathrm{LiCO}_{2}$ & $4.1^{12}$ & 3.51 & 3.53 & 0.59 & 0.57 \\
\hline 9 & $\mathrm{TiS}_{2} \longleftrightarrow \mathrm{LiTiS}_{2}$ & $2.1^{13}$ & 1.85 & 1.95 & 0.25 & 0.15 \\
\hline 10 & $\mathrm{NiPO}_{4} \longleftrightarrow \mathrm{LiNiPO}_{4}$ & $5.3^{14}$ & 4.62 & 4.54 & 0.68 & 0.76 \\
\hline 11 & $\mathrm{~V}_{2}\left(\mathrm{PO}_{4}\right)_{3} \longleftrightarrow \mathrm{Li}_{3} \mathrm{~V}_{2}\left(\mathrm{PO}_{4}\right)_{3}$ & $3.8^{15}$ & 3.85 & 3.58 & 0.05 & 0.22 \\
\hline 12 & $\mathrm{CoO}_{2} \longleftrightarrow \mathrm{NaCoO}_{2}$ & $2.8^{16}$ & 2.87 & 2.90 & 0.07 & 0.1 \\
\hline 13 & $\mathrm{NiO}_{2} \longleftrightarrow \mathrm{NaNiO}_{2}$ & $3.0^{17}$ & 3.13 & 3.07 & 0.13 & 0.07 \\
\hline 14 & $\mathrm{TiO}_{2} \longleftrightarrow \mathrm{NaTiO}_{2}$ & $1.5^{18}$ & 1.18 & 1.03 & 0.32 & 0.47 \\
\hline 15 & $\mathrm{FePO}_{4} \longleftrightarrow \mathrm{NaFePO}_{4}$ & $3^{19}$ & 3.01 & 3.00 & 0.01 & 0.00 \\
\hline 16 & $\mathrm{MnO}_{2} \longleftrightarrow \mathrm{NaMnO}_{2}$ & $3.1^{20}$ & 2.74 & 2.73 & 0.36 & 0.37 \\
\hline 17 & $\mathrm{MnV}\left(\mathrm{PO}_{4}\right)_{3} \longleftrightarrow \mathrm{Na}_{4} \mathrm{MnV}\left(\mathrm{PO}_{4}\right)_{3}$ & $3.5^{21}$ & 3.48 & 3.55 & 0.02 & 0.05 \\
\hline 18 & $\mathrm{Na}_{0.3} \mathrm{Fe}_{0.5} \mathrm{Co}_{0.5} \mathrm{O}_{2} \longleftrightarrow \mathrm{NaFe}_{0.5} \mathrm{Co}_{0.5} \mathrm{O}_{2}$ & $3.25^{22}$ & 3.22 & 2.82 & 0.03 & 0.43 \\
\hline 19 & $\mathrm{Na}_{2} \mathrm{Mn}_{3} \mathrm{O}_{7} \longleftrightarrow \mathrm{K}_{1.6} \mathrm{Na}_{2} \mathrm{Mn}_{3} \mathrm{O}_{7}$ & $2.1^{23}$ & 2.68 & 2.71 & 0.58 & 0.61 \\
\hline \multicolumn{5}{|c|}{ Average absolute deviation } & 0.29 & 0.31 \\
\hline
\end{tabular}




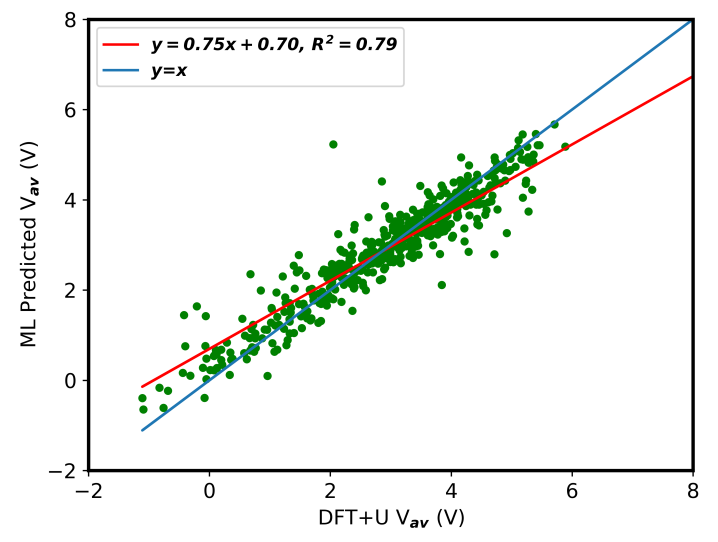

(a)

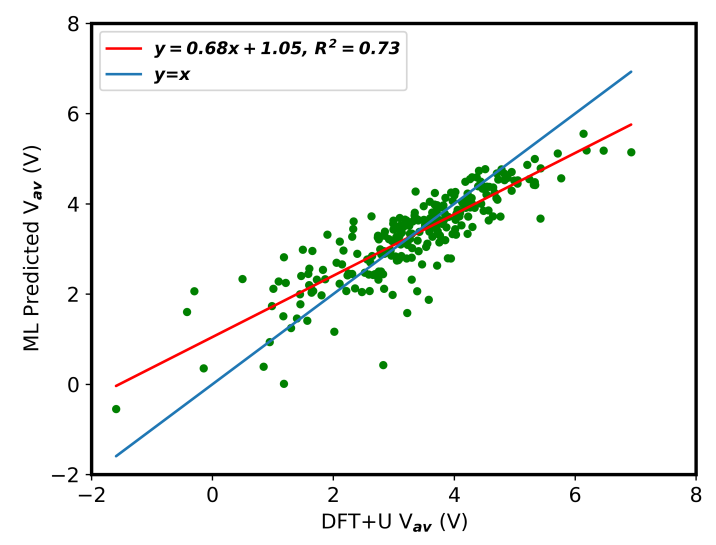

(c)

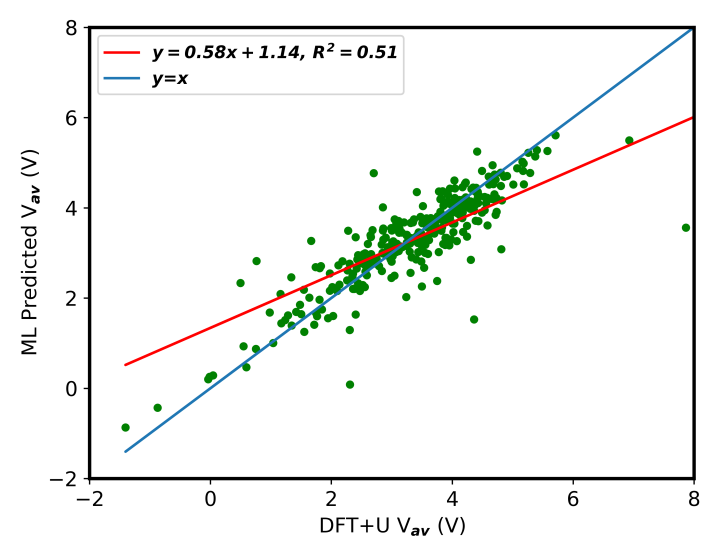

(e)

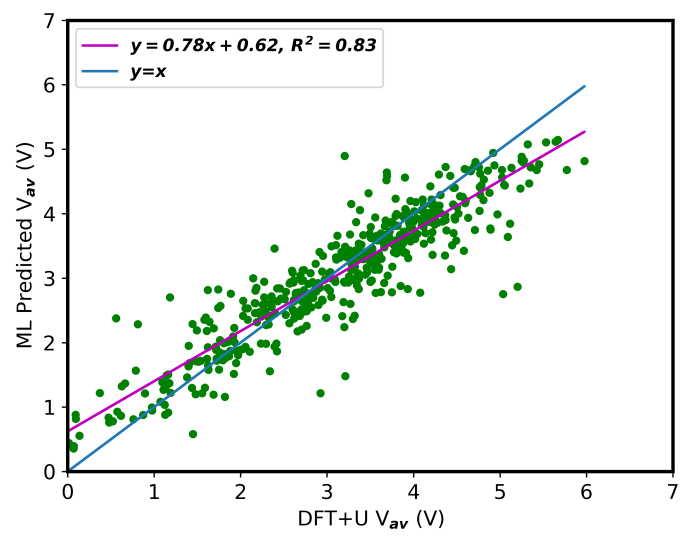

(b)

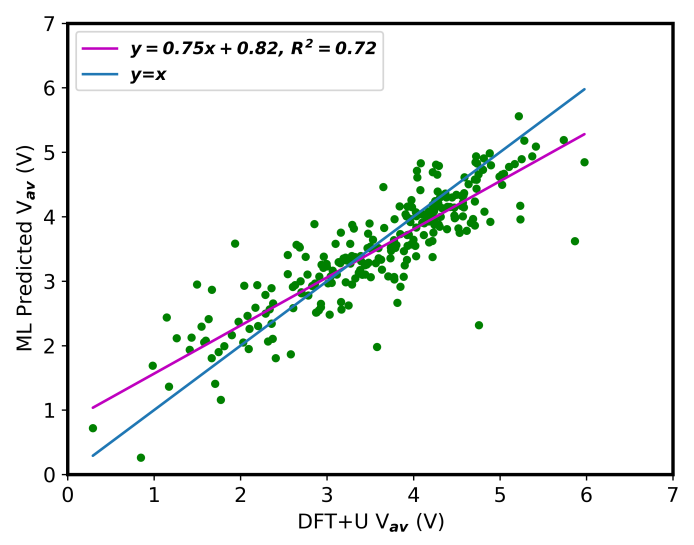

(d)

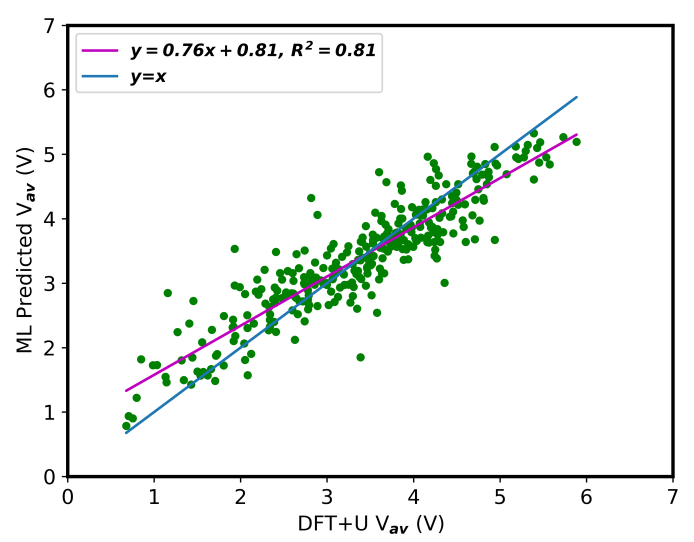

(f)

Figure S1: Test set $\mathrm{V}_{a v}$ values predicted by ML versus the corresponding $\mathrm{DFT}+\mathrm{U}$ values. (a), (c), and (e) are obtained using models trained (and tested) on the full All, Alkali, and Li training (testing) sets, respectively. (b), (d), and (f) are obtained using the models trained (and tested) on All, Alkali, and Li training (testing) sets, respectively, after removing the underrepresented instances from the data. 


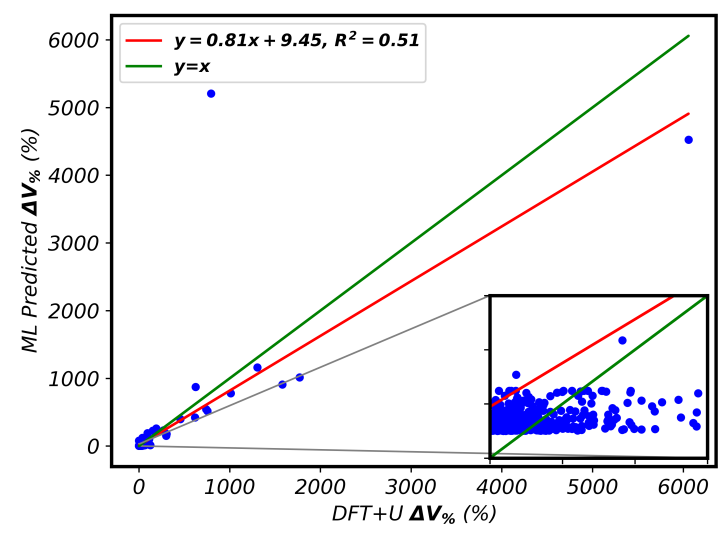

(a)

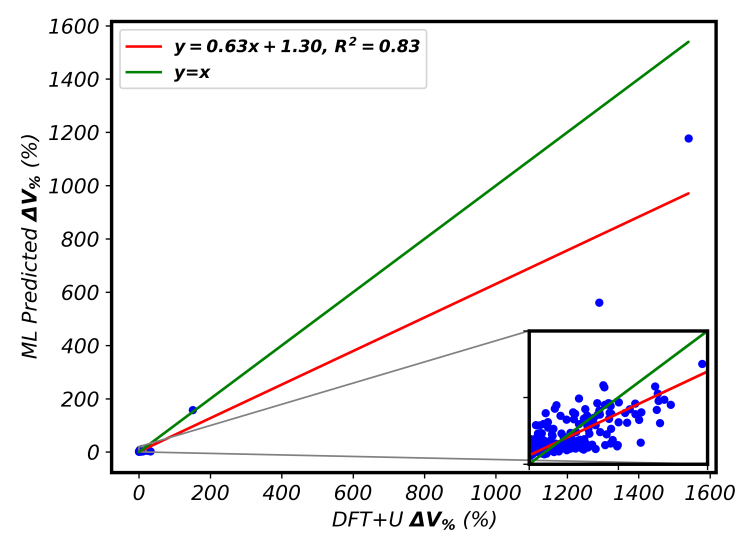

(c)

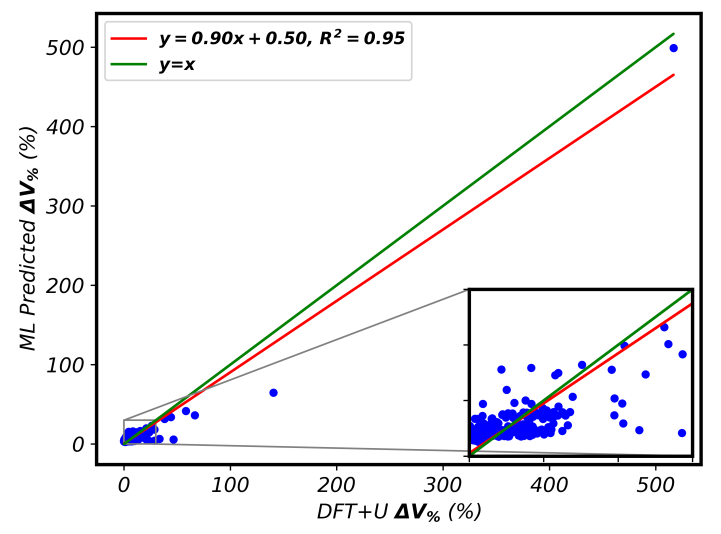

(e)

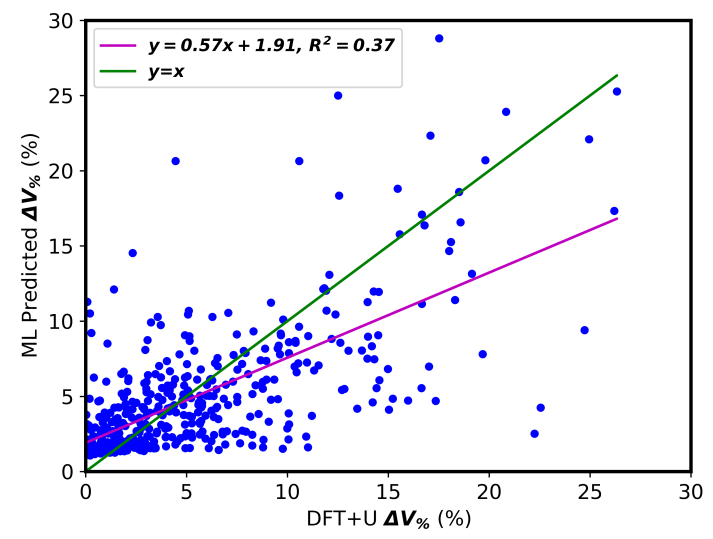

(b)

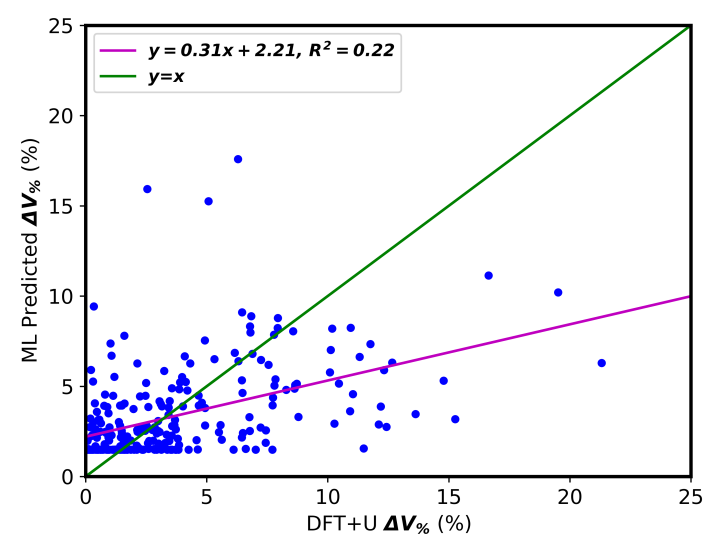

(d)

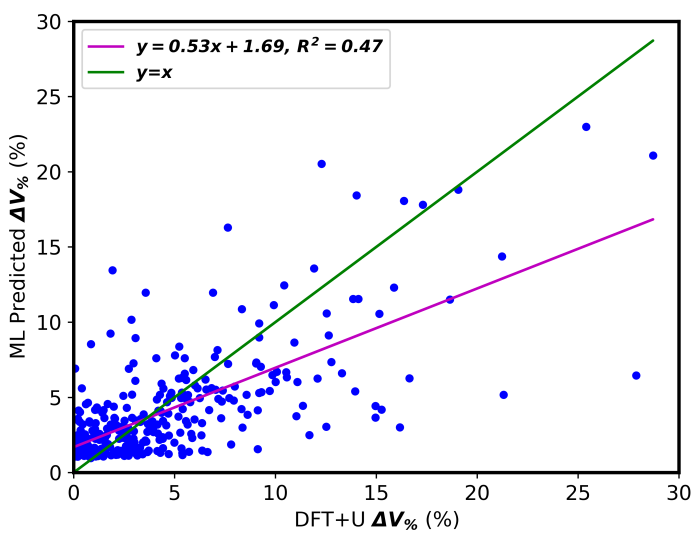

(f)

Figure S2: Test set values of $\Delta V_{\%}$ predicted by ML versus the corresponding DFT $+\mathrm{U}$ values. (a), (c), and (e) are obtained using models trained (and tested) on the full All, Alkali, and Li training (testing) sets, respectively. (b), (d), and (f) are obtained using the models trained (and tested) on All, Alkali, and Li training (testing) sets, respectively, after removing the underrepresented instances from the data. For (a), (c) and (e), the insets in the bottom right represent the zoom-in of the respective marked areas. 


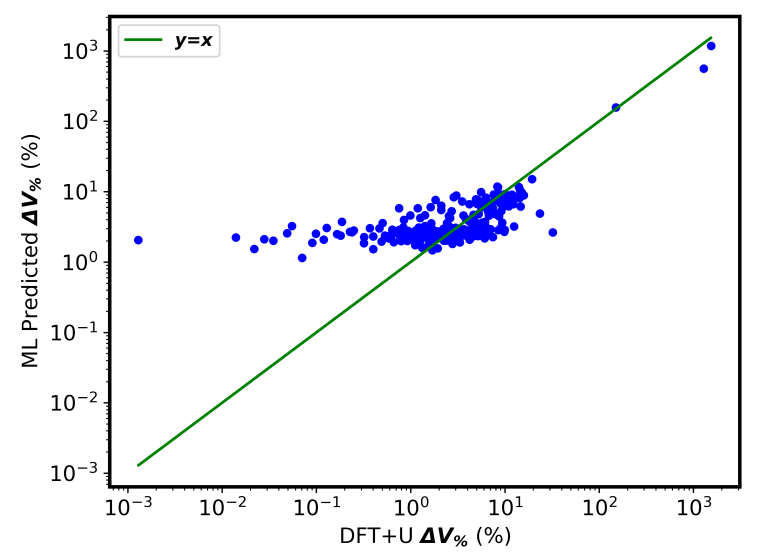

(a)

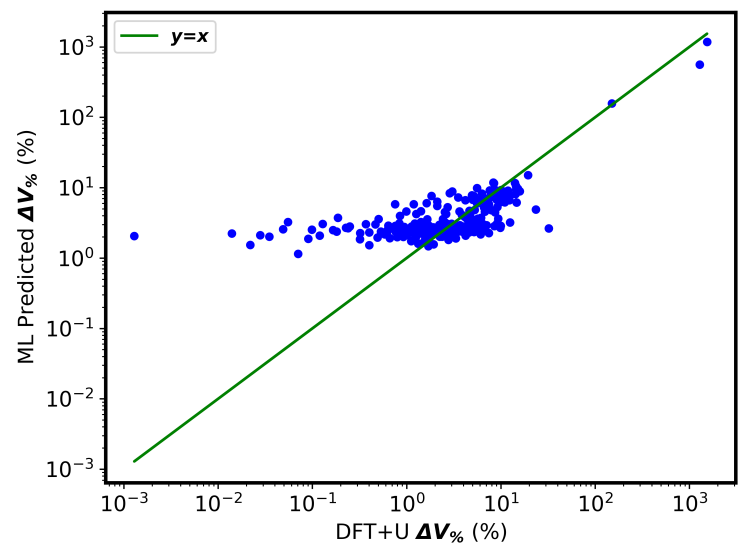

(b)

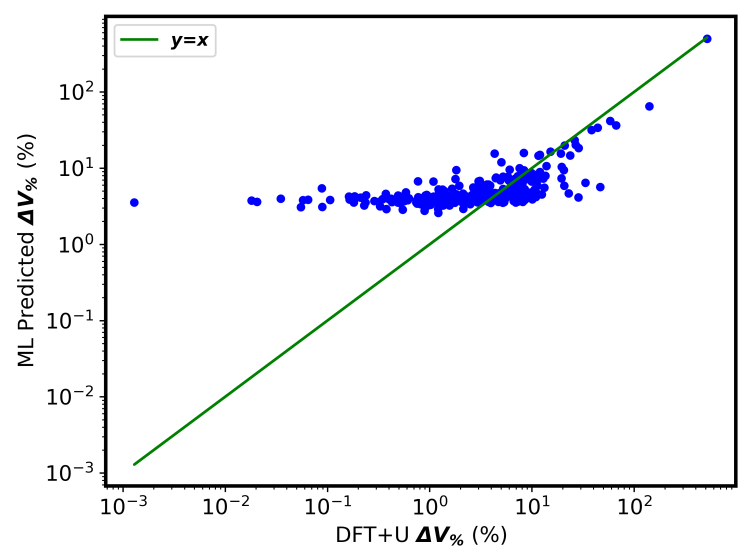

(c)

Figure S3: Test set values of $\Delta V_{\%}$ predicted by ML versus the corresponding $\mathrm{DFT}+\mathrm{U}$ values. (a), (b), and (c) are obtained using models trained (and tested) on the full All, Alkali, and Li training (testing) sets, respectively. 


\section{New Na- and Li-ion Electrodes}

Table S5: Additional results on Na electrodes from Table 5 of main text. Theoretical specific capacity of reactions $(c)$ obtained from reaction stoichiometries considering the active $\mathrm{Na}$ ion mass and $1 e^{-}$transfer from the $\mathrm{Na}$ ion. $\Delta V_{\%}$ and $\mathrm{V}_{a v}$ values predicted by ML models trained with the alkali-ions data set and first-principles DFT+U calculations (Calc.). Machine learning results are obtained from models trained after removing underrepresented instances $(\mathrm{ML})$, and including them $\left(\mathrm{ML}^{*}\right)$ in the data, respectively.

\begin{tabular}{|c|c|c|c|c|c|c|c|c|}
\hline \multirow{2}{*}{\multicolumn{2}{|c|}{ Reaction }} & \multirow{2}{*}{$\frac{C}{(\mathrm{mAh} / \mathrm{g})}$} & \multicolumn{3}{|c|}{$\Delta V_{\%}$} & \multicolumn{3}{|c|}{$\mathbf{V}_{a v}(\mathbf{V})$} \\
\hline & & & ML & $\mathrm{ML}^{*}$ & Calc. & $\overline{\mathrm{ML}}$ & ML* & Calc. \\
\hline 1 & $\mathrm{NaMn}_{3} \mathrm{OF}_{8} \longleftrightarrow \mathrm{Na}_{4} \mathrm{Mn}_{3} \mathrm{OF}_{8}$ & 189 & 5.0 & 5.6 & 26.0 & 3.43 & 3.2 & 3.15 \\
\hline 2 & $\mathrm{Na}\left(\mathrm{CoO}_{2}\right)_{2} \longleftrightarrow \mathrm{Na}_{2} \mathrm{CoO}_{2}$ & 294 & 5.7 & 4.1 & 33.2 & 2.37 & 2.74 & 1.24 \\
\hline 3 & $\mathrm{NaNbTe}_{2} \mathrm{WO}_{12} \longleftrightarrow \mathrm{Na}_{4} \mathrm{NbTe}_{2} \mathrm{WO}_{12}$ & 99 & 4.4 & 4.3 & 15.2 & 1.92 & 3.25 & 3.1 \\
\hline 4 & $\mathrm{NaCuF}_{4} \longleftrightarrow \mathrm{Na}_{2} \mathrm{CuF}_{4}$ & 144 & 2.7 & 11.8 & 20.5 & 4.52 & 4.01 & 4.31 \\
\hline 5 & $\mathrm{Na}_{2} \mathrm{CrO}_{4} \longleftrightarrow \mathrm{Na}_{4} \mathrm{CrO}_{4}$ & 258 & 7.5 & 5.6 & 2.2 & 2.18 & 3.07 & 1.46 \\
\hline 6 & $\mathrm{NaMn}_{2} \mathrm{~F}_{9} \longleftrightarrow \mathrm{Na}_{2} \mathrm{Mn}_{2} \mathrm{~F}_{9}$ & 153 & 3.0 & 7.1 & 14.2 & 3.54 & 3.67 & 4.35 \\
\hline 7 & $\mathrm{Mn}_{3} \mathrm{P}_{6} \mathrm{WO}_{24} \longleftrightarrow \mathrm{Na}_{6} \mathrm{Mn}_{3} \mathrm{P}_{6} \mathrm{WO}_{24}$ & 152 & 4.9 & 6.9 & 25.5 & 2.52 & 3.50 & 3.47 \\
\hline 8 & $\mathrm{CrWO}_{6} \longleftrightarrow \mathrm{Na}_{2} \mathrm{CrWO}_{6}$ & 142 & 2.6 & 4.9 & 3.5 & 2.80 & 3.10 & 3.75 \\
\hline 9 & $\mathrm{NaNbF}_{6} \longleftrightarrow \mathrm{Na}_{3} \mathrm{NbF}_{6}$ & 194 & 4.4 & 4.1 & 1.6 & 1.88 & 2.56 & 3.31 \\
\hline 10 & $\mathrm{NaVTe}\left(\mathrm{WO}_{6}\right)_{2} \longleftrightarrow \mathrm{Na}_{4} \mathrm{VTe}\left(\mathrm{WO}_{6}\right)_{2}$ & 97 & 3.4 & 4.5 & 13.8 & 1.77 & 3.20 & 2.90 \\
\hline 11 & $\mathrm{NaSbF}_{6} \longleftrightarrow \mathrm{Na}_{3} \mathrm{SbF}_{6}$ & 176 & 2.4 & 11.3 & 15.5 & 4.34 & 3.79 & 2.07 \\
\hline 12 & $\mathrm{NaVOF}_{11} \longleftrightarrow \mathrm{Na}_{4} \mathrm{VOF}_{11}$ & 171 & 8.0 & 3.7 & 1.7 & 1.92 & 3.83 & 3.50 \\
\hline $13^{*}$ & $\mathrm{NaV}_{2} \mathrm{O}_{4} \longleftrightarrow \mathrm{Na}_{2} \mathrm{VO}_{2}$ & 312 & 4.9 & 3.3 & 42.3 & 2.26 & 2.85 & -0.35 \\
\hline 14 & $\mathrm{NaV}_{2} \mathrm{OF}_{7} \longleftrightarrow \mathrm{Na}_{3} \mathrm{~V}_{2} \mathrm{OF}_{7}$ & 168 & 7.0 & 3.4 & 19.5 & 2.03 & 3.76 & 3.11 \\
\hline 15 & $\mathrm{CrPO}_{4} \mathrm{~F} \longleftrightarrow \mathrm{Na}_{3} \mathrm{Cr}_{2} \mathrm{P}_{2}\left(\mathrm{O}_{4} \mathrm{~F}\right)_{2}$ & 201 & 3.7 & 4.1 & 22.1 & 5.04 & 3.75 & 3.26 \\
\hline 16 & $\mathrm{NbO}_{2} \mathrm{~F} \longleftrightarrow \mathrm{NaNbO}_{2} \mathrm{~F}$ & 158 & 7.4 & 3.1 & 3.2 & 2.82 & 3.31 & 1.36 \\
\hline 17 & $\mathrm{TiCrO}_{4} \longleftrightarrow \mathrm{Na}_{2} \mathrm{TiCrO}_{4}$ & 255 & 4.7 & 2.4 & 27.0 & 3.54 & 3.31 & 1.54 \\
\hline 18 & $\mathrm{NaTiV}_{3} \mathrm{O}_{10} \longleftrightarrow \mathrm{Na}_{4} \mathrm{TiV}_{3} \mathrm{O}_{10}$ & 178 & 2.9 & 2.4 & 1.1 & 1.96 & 3.00 & 2.47 \\
\hline 19 & $\mathrm{Mn}_{2} \mathrm{CrO}_{6} \longleftrightarrow \mathrm{Na}_{3} \mathrm{Mn}_{2} \mathrm{CrO}_{6}$ & 246 & 9.1 & 2.9 & 25.0 & 3.33 & 2.95 & 2.73 \\
\hline 20 & $\mathrm{NaMn}\left(\mathrm{PO}_{4}\right)_{2} \longleftrightarrow \mathrm{Na}_{3} \mathrm{Mn}\left(\mathrm{PO}_{4}\right)_{2}$ & 171 & 3.8 & 3.0 & 23.6 & 2.67 & 3.28 & 4.32 \\
\hline 21 & $\mathrm{NaMn}_{8} \mathrm{O}_{16} \longleftrightarrow \mathrm{Na}_{3} \mathrm{Mn}_{4} \mathrm{O}_{8}$ & 161 & 1.7 & 3.6 & 24.1 & 2.59 & 2.99 & 1.98 \\
\hline 22 & $\mathrm{VF}_{5} \longleftrightarrow \mathrm{Na}_{2} \mathrm{VF}_{5}$ & 279 & 17.1 & 6.4 & 11.9 & 3.29 & 3.61 & 4.01 \\
\hline
\end{tabular}

* While ML predicts a voltage of about 2.3-2.9 $\mathrm{V}$ for this material, DFT+U predicts a negative value indicating that it will not work as a Na-ion electrode.

Note: specific energy density (in $\mathrm{Wh} / \mathrm{kg}$ ) is obtained as the product of $c$ and $\mathrm{V}_{a v}$.

New L-ion electrodes taken from instances present in the Na-ion database with not data in the corresponding L-ion data set were explored by replacing $\mathrm{Na}$ by Li atoms. The corresponding ML results are shown in Table S7. 
Table S6: Crystal Symmetry of the Na-ion Electrodes

\begin{tabular}{|c|c|c|c|}
\hline & Reaction & Lattice System & Space Group \\
\hline 1 & $\mathrm{NaMn}_{3} \mathrm{OF}_{8} \longleftrightarrow \mathrm{Na}_{4} \mathrm{Mn}_{3} \mathrm{OF}_{8}$ & Monoclinic & $\mathrm{Pc}$ \\
\hline 2 & $\mathrm{Na}\left(\mathrm{CoO}_{2}\right)_{2} \longleftrightarrow \mathrm{Na}_{2} \mathrm{CoO}_{2}$ & Trigonal & P $\overline{3} \mathrm{~m} 1$ \\
\hline 3 & $\mathrm{NaNbTe}_{2} \mathrm{WO}_{12} \longleftrightarrow \mathrm{Na}_{4} \mathrm{NbTe}_{2} \mathrm{WO}_{12}$ & Triclinic & P1 \\
\hline 4 & $\mathrm{NaCuF}_{4} \longleftrightarrow \mathrm{Na}_{2} \mathrm{CuF}_{4}$ & Monoclinic & $\mathrm{P} 22_{1} / \mathrm{m}$ \\
\hline 5 & $\mathrm{Na}_{2} \mathrm{CrO}_{4} \longleftrightarrow \mathrm{Na}_{4} \mathrm{CrO}_{4}$ & Triclinic & $\mathrm{P} \overline{1}$ \\
\hline 6 & $\mathrm{NaMn}_{2} \mathrm{~F}_{9} \longleftrightarrow \mathrm{Na}_{2} \mathrm{Mn}_{2} \mathrm{~F}_{9}$ & Triclinic & P1 \\
\hline 7 & $\mathrm{Mn}_{3} \mathrm{P}_{6} \mathrm{WO}_{24} \longleftrightarrow \mathrm{Na}_{6} \mathrm{Mn}_{3} \mathrm{P}_{6} \mathrm{WO}_{24}$ & Trigonal & R3 \\
\hline 8 & $\mathrm{CrWO}_{6} \longleftrightarrow \mathrm{Na}_{2} \mathrm{CrWO}_{6}$ & Triclinic & P1 \\
\hline 9 & $\mathrm{NaNbF}_{6} \longleftrightarrow \mathrm{Na}_{3} \mathrm{NbF}_{6}$ & Trigonal & $\mathrm{R} \overline{3}$ \\
\hline 10 & $\operatorname{NaVTe}\left(\mathrm{WO}_{6}\right)_{2} \longleftrightarrow \mathrm{Na}_{4} \mathrm{VTe}\left(\mathrm{WO}_{6}\right)_{2}$ & Triclinic & P1 \\
\hline 11 & $\mathrm{NaSbF}_{6} \longleftrightarrow \mathrm{Na}_{3} \mathrm{SbF}_{6}$ & Trigonal & $\mathrm{R} \overline{3}$ \\
\hline 12 & $\mathrm{NaVOF}_{11} \longleftrightarrow \mathrm{Na}_{4} \mathrm{VOF}_{11}$ & Triclinic & $\mathrm{P} \overline{1}$ \\
\hline 13 & $\mathrm{NaV}_{2} \mathrm{O}_{4} \longleftrightarrow \mathrm{Na}_{2} \mathrm{VO}_{2}$ & Monoclinic & $\mathrm{P} 2{ }_{1} / \mathrm{m}$ \\
\hline 14 & $\mathrm{NaV}_{2} \mathrm{OF}_{7} \longleftrightarrow \mathrm{Na}_{3} \mathrm{~V}_{2} \mathrm{OF}_{7}$ & Triclinic & P1 \\
\hline 15 & $\mathrm{CrPO}_{4} \mathrm{~F} \longleftrightarrow \mathrm{Na}_{3} \mathrm{Cr}_{2} \mathrm{P}_{2}\left(\mathrm{O}_{4} \mathrm{~F}\right)_{2}$ & Triclinic & $\mathrm{P} \overline{1}$ \\
\hline 16 & $\mathrm{NbO}_{2} \mathrm{~F} \longleftrightarrow \mathrm{NaNbO}_{2} \mathrm{~F}$ & Monoclinic & $\mathrm{C} 2 / \mathrm{c}$ \\
\hline 17 & $\mathrm{TiCrO}_{4} \longleftrightarrow \mathrm{Na}_{2} \mathrm{TiCrO}_{4}$ & Tetragonal & $\mathrm{I} \overline{4} \mathrm{~m} 2$ \\
\hline 18 & $\mathrm{NaTiV}_{3} \mathrm{O}_{10} \longleftrightarrow \mathrm{Na}_{4} \mathrm{TiV}_{3} \mathrm{O}_{10}$ & Monoclinic & $\mathrm{C} 2$ \\
\hline 19 & $\mathrm{Mn}_{2} \mathrm{CrO}_{6} \longleftrightarrow \mathrm{Na}_{3} \mathrm{Mn}_{2} \mathrm{CrO}_{6}$ & Monoclinic & $\mathrm{C} 2 / \mathrm{m}$ \\
\hline 20 & $\mathrm{NaMn}\left(\mathrm{PO}_{4}\right)_{2} \longleftrightarrow \mathrm{Na}_{3} \mathrm{Mn}\left(\mathrm{PO}_{4}\right)_{2}$ & Monoclinic & $\mathrm{P} 2{ }_{1} / \mathrm{c}$ \\
\hline 21 & $\mathrm{NaMn}_{8} \mathrm{O}_{16} \longleftrightarrow \mathrm{Na}_{3} \mathrm{Mn}_{4} \mathrm{O}_{8}$ & Triclinic & P1 \\
\hline 22 & $\mathrm{VF}_{5} \longleftrightarrow \mathrm{Na}_{2} \mathrm{VF}_{5}$ & Triclinic & P1 \\
\hline
\end{tabular}

Table S7: Theoretical specific capacity of reactions $(c)$ obtained from reaction stoichiometries considering the active $\mathrm{Li}$ ion mass and $1 e^{-}$transfer from the Li ion. $\Delta V_{\%}$ and $\mathrm{V}_{a v}$ values predicted by ML models trained with the alkali-ions data set and first-principles DFT $+\mathrm{U}$ calculations (Calc.). Machine learning results are obtained from models trained after removing underrepresented instances $(\mathrm{ML})$, and including them $\left(\mathrm{ML}^{*}\right)$ in the data, respectively.

\begin{tabular}{l|c|c|ccc|ccc}
\hline \multirow{2}{*}{\multicolumn{2}{c|}{ Reaction }} & $c$ & \multicolumn{3}{|c|}{$\Delta V_{\%}$} & \multicolumn{3}{c}{$\mathbf{V}_{a v}(\mathbf{V})$} \\
\cline { 3 - 9 } & & $(\mathrm{mAh} / \mathrm{g})$ & ML & ML* & Calc. & ML & ML* & Calc. \\
\hline 1 & $\mathrm{NbO}_{3} \longleftrightarrow \mathrm{LiNbO}_{3}$ & 181 & 1.2 & 4.8 & 12.9 & 4.05 & 4.01 & 4.69 \\
2 & $\mathrm{Li}_{10} \mathrm{Mn}_{2} \mathrm{O}_{9} \longleftrightarrow \mathrm{Li}_{14} \mathrm{Mn}_{2} \mathrm{O}_{9}$ & 305 & 4.0 & 4.6 & 7.5 & 3.86 & 1.92 & 2.26 \\
3 & $\mathrm{CrF}_{6} \longleftrightarrow \mathrm{LiCrF}_{6}$ & 155 & 2.2 & 4.9 & 13.6 & 5.63 & 5.55 & 5.83 \\
4 & $\left.\mathrm{~V}\left(\mathrm{NF}_{3}\right)_{2} \longleftrightarrow \mathrm{LiV}_{2} \mathrm{NF}_{3}\right)_{2}$ & 134 & 8.2 & 6.7 & 2.3 & 4.37 & 5.44 & 6.10 \\
5 & $\mathrm{SbO}_{3} \longleftrightarrow \mathrm{LiSbO}_{3}$ & 152 & 2.6 & 9.5 & 4.7 & 4.61 & 4.68 & 4.72 \\
$6^{*}$ & $\mathrm{TiF}_{3} \longleftrightarrow \mathrm{LiTiF}_{3}$ & 240 & 4.8 & 9.3 & 11.9 & 1.82 & 0.82 & $-0.85^{*}$ \\
7 & $\mathrm{LiGePCO}_{7} \longleftrightarrow \mathrm{Li}_{3} \mathrm{GePCO}_{7}$ & 216 & 6.3 & 10.2 & 8.9 & 3.13 & 2.50 & 2.49 \\
\hline
\end{tabular}

* While ML predicts a voltage of about $0.82-1.82 \mathrm{~V}$ for this material, DFT+U predicts a negative value indicating that it will not work as a Li-ion electrode. Note: Specific energy density (in $\mathrm{Wh} / \mathrm{kg}$ ) is obtained as the product of $c$ and $\mathrm{V}_{a v}$. 


\section{References}

(1) Ward, L.; Dunn, A.; Faghaninia, A.; Zimmermann, N. E. R.; Bajaj, S.; Wang, Q.; Montoya, J. H.; Chen, J.; Bystrom, K.; Dylla, M.; Chard, K.; Asta, P. K., M. and; Snyder, G. J.; Foster, I.; Jain, A. Matminer: An Open Source Toolkit for Materials Data Mining. Comput. Mater. Sci. 2018, 152, 60-69.

(2) Ward, L.; Agrawal, A.; Choudhary, A.; Wolverton, C. A General-purpose Machine Learning Framework for Predicting Properties of Inorganic Materials. npj Comput. Mater. 2016, 2, 1-7.

(3) Meredig, B.; Agrawal, A.; Kirklin, S.; Saal, J. E.; Doak, J.; Thompson, A.; Zhang, K.; Choudhary, A.; Wolverton, C. Combinatorial Screening for New Materials in Unconstrained Composition Space with Machine Learning. Phys. Rev. B Condens. Matter 2014, 89, 094104.

(4) Butler, M.; Ginley, D. Prediction of Flatband Potentials at Semiconductor-electrolyte Interfaces from Atomic Electronegativities. J. Electrochem. Soc. 1978, 125, 228.

(5) Deml, A. M.; O’Hayre, R.; Wolverton, C.; Stevanović, V. Predicting Density Functional Theory Total Energies and Enthalpies of Formation of Metal-nonmetal Compounds by Linear Regression. Phys. Rev. B Condens. Matter 2016, 93, 085142.

(6) Li, G.; Azuma, H.; Tohda, M. $\mathrm{LiMnPO}_{4}$ as the Cathode for Lithium Batteries. Electrochem. Solid-State Lett. 2002, 5, A135.

(7) Yamada, A.; Chung, S.-C.; Hinokuma, K. Optimized $\mathrm{LiFePO}_{4}$ for Lithium Battery Cathodes. J. Electrochem. Soc. 2001, 148, A224.

(8) Amine, K.; Yasuda, H.; Yamachi, M. Olivine $\mathrm{LiCoPO}_{4}$ as 4.8V Electrode Material for Lithium Batteries. Electrochem. Solid-State Lett. 2000, 3, 178. 
(9) Choi, S.; Manthiram, A. Synthesis and Electrochemical Properties of $\mathrm{LiCo}_{2} \mathrm{O}_{4} \mathrm{Spinel}$ Cathodes. J. Electrochem. Soc. 2002, 149, A162.

(10) Delmas, C.; Menetrier, M.; Croguennec, L.; Levasseur, S.; Peres, J.; Pouillerie, C.; Prado, G.; Fournes, L.; Weill, F. Lithium Batteries: A New Tool in Solid State Chemistry. Int. J. Inorg. Mater 1999, 1, 11-19.

(11) Ohzuku, T.; Ueda, A. Why Transition Metal (Di) Oxides are the Most Attractive Materials for Batteries. Solid State Ion. 1994, 69, 201-211.

(12) Amatucci, G.; Tarascon, J.; Klein, L. $\mathrm{CoO}_{2}$, the End Member of the $\mathrm{Li}_{x} \mathrm{CoO}_{2}$ Solid Solution. J. Electrochem. Soc. 1996, 143, 1114.

(13) Whittingham, M. S. Electrical Energy Storage and Intercalation Chemistry. Science 1976, 192, 1126-1127.

(14) Wolfenstine, J.; Allen, J. Ni3+/Ni2+ Redox Potential in $\mathrm{LiNiPO}_{4}$. J. Power Sources 2005, 142, 389-390.

(15) Gaubicher, J.; Wurm, C.; Goward, G.; Masquelier, C.; Nazar, L. Rhombohedral form of Li3V2 $\left(\mathrm{PO}_{4}\right)_{3}$ as a Cathode in Li-ion Batteries. Chem. Mater. 2000, 12, 3240-3242.

(16) Delmas, C.; Braconnier, J.-J.; Fouassier, C.; Hagenmuller, P. Electrochemical Intercalation of Sodium in $\mathrm{Na}_{x} \mathrm{CoO}_{2}$ Bronzes. Solid State Ion. 1981, 3, 165-169.

(17) Braconnier, J.; Delmas, C.; Hagenmuller, P. Etude par Desintercalation electrochimique des Systemes $\mathrm{NaxCrO}_{2}$ et $\mathrm{NaxNiO}_{2}$. Mater. Res. Bull. 1982, 17, 993-1000.

(18) Maazaz, A.; Delmas, C.; Hagenmuller, P. A Study of the $\mathrm{Na}_{x} \mathrm{TiO}_{2}$ System by Electrochemical Deintercalation. J. Incl. Phenom. 1983, 1, 45-51.

(19) Moreau, P.; Guyomard, D.; Gaubicher, J.; Boucher, F. Structure and Stability of Sodium Intercalated Phases in Olivine $\mathrm{FePO}_{4}$. Chem. Mater. 2010, 22, 4126-4128. 
(20) Billaud, J.; Clément, R. J.; Armstrong, A. R.; Canales-Vázquez, J.; Rozier, P.; Grey, C. P.; Bruce, P. G. $\beta-\mathrm{NaMnO}_{2}$ : a High-performance Cathode for Sodium-ion Batteries. J. Am. Chem. Soc. 2014, 136, 17243-17248.

(21) Nisar, U.; Shakoor, R.; Essehli, R.; Amin, R.; Orayech, B.; Ahmad, Z.; Kumar, P. R.; Kahraman, R.; Al-Qaradawi, S.; Soliman, A. Sodium Intercalation/De-intercalation Mechanism in $\mathrm{Na}_{4} \mathrm{MnV}\left(\mathrm{PO}_{4}\right)_{3}$ Cathode Materials. Electrochim. Acta 2018, 292, 98106.

(22) Yoshida, H.; Yabuuchi, N.; Komaba, S. $\mathrm{NaFe}_{0.5} \mathrm{Co}_{0.5} \mathrm{O}_{2}$ as High Energy and Power Positive Electrode for Na-ion Batteries. Electrochem. commun 2013, 34, 60-63.

(23) Sada, K.; Senthilkumar, B.; Barpanda, P. Potassium-ion Intercalation Mechanism in Layered $\mathrm{Na}_{2} \mathrm{Mn}_{3} \mathrm{O}_{7}$. ACS Appl. Energy Mater. 2018, 1, 5410-5416. 